\title{
Preliminary evaluation of postoperative radiotherapy with small T-shaped field in thoracic esophageal squamous cell carcinoma
}

\author{
Qi Zhao", Jiaxing Zhu\#, Yandong Liu, Songbing Qin, Juying Zhou \\ Department of Radiation Oncology, The First Affiliated Hospital of Soochow University, Suzhou, China \\ Contributions: (I) Conception and design: J Zhou, S Qin, Q Zhao; (II) Administrative support: J Zhu; (III) Provision of study materials or patients: \\ Y Liu; (IV) Collection and assembly of data: J Zhu; (V) Data analysis and interpretation: Q Zhao, J Zhu; (VI) Manuscript writing: All authors; (VII) \\ Final approval of manuscript: All authors. \\ \#These authors contributed equally to this work. \\ Correspondence to: Juying Zhou; Songbing Qin. Department of Radiation Oncology, The First Affiliated Hospital of Soochow University, Shi Zi Street \\ 188, Suzhou 215006, China. Email: zhoujy19652021@163.com; qin92244@163.com.
}

Background: The optimal extent of clinical target volume (CTV) for postoperative radiotherapy in complete resection thoracic esophageal squamous cell carcinoma (ESCC) patients remains controversial. This study aimed to evaluate the primary outcome of postoperative radiotherapy with small T-shaped field encompassing the tumor bed, positive lymph node areas, bilateral supraclavicular areas, and upper and middle mediastinal areas.

Methods: A total of 96 thoracic ESCC patients were enrolled, with 49 and 47 cases in the small T-shaped field group and tumor bed field group, respectively. All of the patients received intensity-modulated radiotherapy (IMRT), and chemotherapy was administrated concurrently or sequentially. The median time of follow-up was 25 (range, 7-47) months.

Results: At the end of the follow-up period, in the small T-shaped field group, 8 (16.3\%) patients had locoregional recurrence (LRR) and 12 (24.5\%) had distant metastasis (DM), while in the tumor bed field group, 15 (31.9\%) patients had LRR and 11 (23.4\%) had DM. Although the rates of LRR and DM were not statistically different, LRR incidence in the mediastinal lymph nodes of the small T-shaped field group was strikingly lower than that of the tumor bed field group. The overall survival (OS) of the small T-shaped field group was higher than that of the tumor bed field group, but the difference was not statistically significance. In addition, we observed grade 2 radiation pneumonitis and grade 2 radiation esophagitis in both groups; all of these side effects were tolerable and controllable, and none of the patients experienced $\geq$ grade 3 pneumonitis, esophagitis, esophageal stricture, or life-threatening hemorrhage.

Conclusions: In conclusion, radiotherapy with small T-shaped field might be a feasible and efficacious postoperative approach for ESCC patients.

Keywords: Esophageal squamous cell carcinoma (ESCC); postoperative radiotherapy; small T-shaped field; intensity-modulated radiotherapy (IMRT)

Submitted Aug 22, 2021. Accepted for publication Oct 21, 2021.

doi: 10.21037/jgo-21-604

View this article at: https://dx.doi.org/10.21037/jgo-21-604

\section{Introduction}

Esophageal carcinoma is one of the most common malignant tumors worldwide, and is the sixth leading cause of cancerrelated mortality (1), with nearly half of all new cases occurring in China (2). Although neoadjuvant therapy is recommended for resectable locally advanced esophageal carcinoma (3), surgical removal usually represents the initial treatment for the most thoracic esophageal squamous cell carcinoma (ESCC) patients in China. However, the therapeutic outcome of surgery alone is not satisfactory, and the recurrence rate is 
as high as $43.3-50.0 \%(4,5)$. Locoregional recurrence (LRR) and distant metastasis (DM) are still the major reasons for postoperative failure. Therefore, postoperative radiotherapy and chemoradiotherapy have been proposed for the treatment of ESCC. Accumulating evidence highlights the improved effects of these postoperative regimens on overall survival (OS) and disease-free survival (DFS) compared with surgery alone in ESCC patients (6-10).

Due to the intricate lymphatic drainage of the esophagus with cervical, mediastinal, and celiac nodes, there is no consensus on the optimal range of postoperative radiotherapy for ESCC $(11,12)$. Lymph node metastasis of ESCC has the characteristics of longitudinal metastasis along the submucosal lymphatic network and skip metastasis, so the standard clinical target volume (CTV) should encompass the tumor bed, bilateral supraclavicular areas, mediastinal lymph nodes, as well as the cardia and left gastric lymph nodes (13-16). Theoretically, the full coverage of sub-clinical areas could reduce the incidence of LRR within the irradiated field; however, severe side effects such as radiation pneumonia should not be neglected in such an extensive range of irradiation, and complications may offset its survival advantage in certain patients. Several different kinds of radiation field designs have been reported for postoperative radiotherapy in ESCC, including the tumor bed alone (17), the bilateral supraclavicular areas and entire mediastinum (18), bilateral supraclavicular areas, entire mediastinum and left gastric lymphatic drainage areas (19), and T-shaped field (bilateral lower neck, bilateral supraclavicular areas and upper middle mediastinum) (20). Related literature reports that such a large range of irradiation fields (standard clinical target areas) often cause severe gastrointestinal and systemic reactions, and many poorly tolerated patients have to suspend treatment or reduce the irradiation dose. Some patients even experience severe radiation pulmonary fibrosis, non-cancerous pericardial effusion, pleural effusion, and gastrointestinal bleeding (13). The CTV was adjusted to a T-shaped field, covering the bilateral supraclavicular area, mediastinum and tumor bed and no longer irradiating the left gastric lymph nodes. Acute and late radiotherapy complications such as gastric bleeding and heart and lung complications are significantly reduced after the use of T-shaped fields (21). In order to optimize the radiation field for ESCC, we designed the small $\mathrm{T}$-shaped field covering the bilateral supraclavicular areas, as well as the upper and middle mediastinal lymphatic drainage areas. Such an irradiation range not only includes the high-risk areas of postoperative recurrence, but also takes the occurrence of adverse reactions into account. Herein, we retrospectively compared the occurrence of LRR and DM, the survival rate, as well as the radiotherapy toxicity between the small T-shaped field and the tumor bed field. Our results suggested the acceptance and feasibility of postoperative radiotherapy with the small T-shaped field for ESCC patients.

We present the following article in accordance with the STROBE reporting checklist (available at https://dx.doi. org/10.21037/jgo-21-604).

\section{Methods}

\section{Subjects}

A retrospective study was performed to analyze 96 thoracic ESCC patients who were underwent a radical esophagectomy in The First Affiliated Hospital of Soochow University (Suzhou, China) from 2016 to 2020 . The subjects included 85 males and 11 females, with an average age of $62.76 \pm 6.78$ years. The number of cases during the study period determined the sample size. Tumor staging was based on the American Joint Committee on Cancer/Union for International Cancer Control tumor-node-metastasis (TNM) staging system ( $8^{\text {th }}$ edition) $(22)$. The TNM stages of the patients were IIB-IVA.

The inclusion criteria were as follows: (I) postoperative pathological examination-confirmed thoracic ESCC with stage T3/T4 or positive nearby lymph node; (II) abdominal B-ultrasound, computed tomography (CT), magnetic resonance imaging (MRI) or positron emission tomographycomputer tomography (PET-CT) revealed no metastasis in the supraclavicular lymph node or abdominal cavity; (III) patients with normal electrocardiogram, urine routine, liver, and renal function tests; and (IV) patients with an Eastern Cooperative Oncology Group (ECOG) performance status (PS) of 0-1.

The exclusion criteria were as follows: (I) patients with residual tumor after surgery; (II) patients with adenocarcinoma or other histologic types; (III) patients with previous malignancies; (IV) patients with distant hematogenous metastasis; and (V) patients with indications of other systemic diseases, such as acute infection, chronic obstructive pulmonary disease, heart failure, acquired immune deficiency syndrome, etc.

\section{Radiotherapy and chemotherapy}

ESCC patients received intensity-modulated radiotherapy (IMRT) 4-6 weeks after radical esophagectomy using 

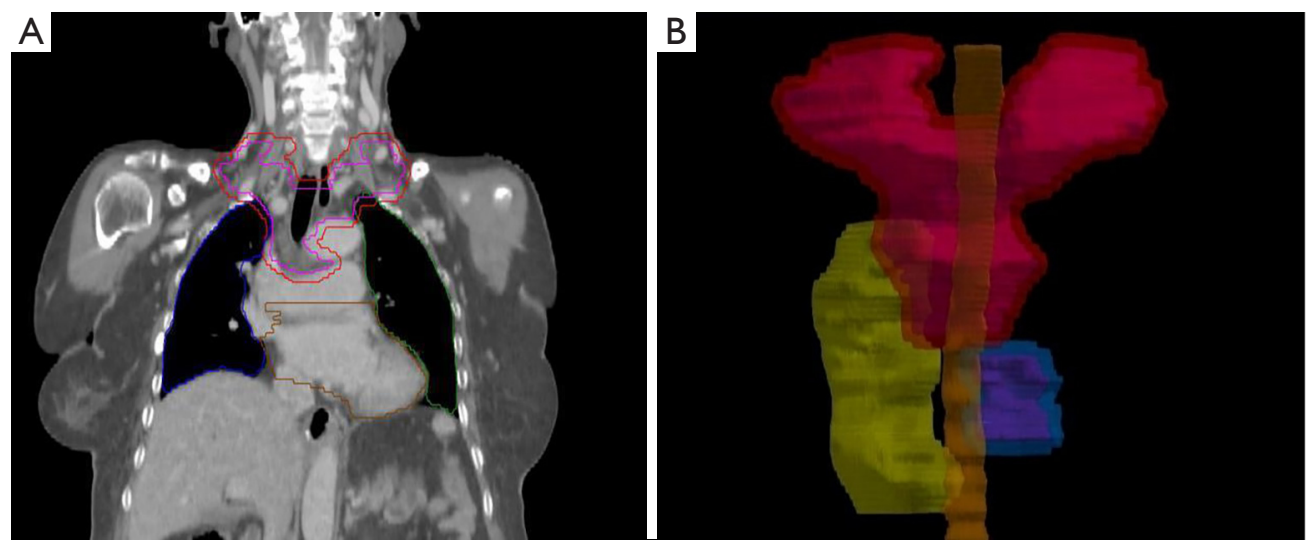

Figure 1 Schematic diagram of the targeted areas. (A) The pink area was the CTV (small T-shaped field). The heart, left lung, and right lung were delineated with brown, blue, and green lines, respectively. (B) The tumor bed were marked with purple. The stomach and spinal cord were delineated with yellow and brown respectively. CTV, clinical target volume.

$6 \mathrm{MeV}$ photons delivered by a linear accelerator (Elekta Synergy). The CTV included the bilateral supraclavicular areas as well as the upper and middle mediastinal lymphatic drainage areas (Figure 1). The total dose was 50.4 Gy/ 28 fractions, irradiated 1.8 Gy per day, 5 days per week. The total dose for the T3/T4 tumor bed and positive lymph node areas gross tumor volume (GTV) was $60.2 \mathrm{~Gy} /$ 28 fractions, irradiated 2.15 Gy per day, 5 days per week. For patients in the small T-shaped field group, both the CTV and GTV were irradiated, whereas for the patients in the tumor bed field group, only GTV was irradiated. At least $95 \%$ of planning target volume (PTV) reaches $100 \%$ of the prescription dose, and $99 \%$ of PTV reaches $95 \%$ of the prescription dose. All of the patients were concurrently or sequentially treated with chemotherapy $\left(135 \mathrm{mg} / \mathrm{m}^{2}\right.$ of paclitaxel on day 1 , and $75 \mathrm{mg} / \mathrm{m}^{2}$ platinum on days 1,2 , and 3 or $80 \mathrm{mg} / \mathrm{m}^{2}$ of nedaplatin on day 2) for $4-6$ cycles.

\section{Follow-up}

All patients were followed-up every $2-3$ months within the first 2 years, and every $4-6$ months thereafter. The follow-up examination included a clinical evaluation, blood biochemistry test, as well as CT of the neck, chest, and upper abdomen. Endoscopy, bone scan, brain magnetic resonance, or PET-CT were performed when necessary. The median time of follow-up was 25 (range, 7-47) months.

\section{Observation indicators}

LRR was defined as regional lymph node relapse, tumor bed, or anastomotic recurrence. DM was defined as metastasis to a distant organ (liver, lung, bone, etc.), or non-regional lymph node metastasis (the abdominal cavity lymph node for upper thoracic esophageal carcinoma or the supraclavicular lymph node for lower thoracic esophageal carcinoma). OS was regarded as the time from the date of initial treatment to death or the last available vital status data. Toxicity was categorized according to the Common Terminology Criteria for Adverse Events (CTCAE) version 4.0.

\section{Statistical analysis}

Statistical analysis was performed using SPSS 25.0 software (IBM SPSS Statistics 25.0). Cumulative survival rates were calculated using Kaplan-Meier survival curves. The Student's $t$-test, Chi-square test, or Fisher's exact test were used to compare the differences between the two groups. All statistical tests were two-sided, and $\mathrm{P}<0.05$ was considered to be statistically significant.

\section{Ethical statement}

The study was conducted in accordance with the Declaration of Helsinki (as revised in 2013). The study was approved by ethics board of The First Affiliated Hospital of Soochow University (No. 2020049) and informed consent was taken from all the patients.

\section{Results}

A total of 96 thoracic ESCC patients were included 
Table 1 Clinicopathological characteristics

\begin{tabular}{|c|c|c|c|c|}
\hline Parameters & T-shape field ( $n=49), n(\%)$ & Tumor bed field ( $n=47), n(\%)$ & Test value & $P$ value \\
\hline \multicolumn{5}{|l|}{ Age (years) } \\
\hline Min & 44 & 49 & & \\
\hline Max & 76 & 75 & & \\
\hline Mean \pm SD & $63.69 \pm 7.01$ & $61.79 \pm 6.46$ & $1.384^{\mathrm{a}}$ & 0.170 \\
\hline Male & $44(89.80)$ & $41(87.23)$ & & \\
\hline Female & $5(10.20)$ & $6(12.77)$ & & \\
\hline Tumor location & & & $0.484^{b}$ & 0.785 \\
\hline Upper & 7 (14.29) & $5(10.64)$ & & \\
\hline T stage & & & $1.422^{\mathrm{c}}$ & 0.526 \\
\hline $\mathrm{T} 1$ & 0 & 0 & & \\
\hline $\mathrm{T} 2$ & $9(18.37)$ & $12(25.53)$ & & \\
\hline T3 & 34 (69.39) & $32(68.09)$ & & \\
\hline $\mathrm{T} 4 \mathrm{a}$ & $6(12.24)$ & $3(6.38)$ & & \\
\hline $\mathrm{N}$ stage & & & $2.679^{c}$ & 0.281 \\
\hline No & $26(53.06)$ & $23(48.94)$ & & \\
\hline N1 & 17 (34.69) & $22(56.81)$ & & \\
\hline IIIA & $6(12.24)$ & $10(21.28)$ & & \\
\hline IIIB & $16(32.65)$ & $16(34.04)$ & & \\
\hline IVA & $1(2.04)$ & 0 & & \\
\hline
\end{tabular}

${ }^{\text {a }}$, Student's $t$-test; ${ }^{\mathrm{b}}$, Chi-square test; ${ }^{\mathrm{c}}$, Fisher's exact test.

in the present study. Four to six weeks after radical esophagectomy, 49 patients were received radiotherapy with small T-shaped field and 47 patients received irradiation of the tumor bed field. The clinicopathological features are summarized in Table 1, and no significant differences were observed between the two groups in terms of age, gender, tumor location, or tumor staging data (all $\mathrm{P}>0.05$ ).

As shown in Table 2, the overall incidence of LRR between the small T-shaped field group $(n=8 / 49,16.3 \%)$ and tumor bed field group ( $n=15 / 47,31.9 \%$ ) were not significantly different $(\mathrm{P}=0.12)$ at the end of follow-up. However, the anatomical distribution of LRR might display distinctive patterns, since the LRR rate in the mediastinal lymph nodes of the tumor bed field group was statistically higher than that of the small T-shaped field group, but not in the other lymph nodes, anastomotic sides, or tumor bed. In addition, the two groups showed equal frequencies of DM, as 12 $(24.5 \%)$ patients in the small T-shaped field group had $\mathrm{DM}$, and $11(23.4 \%)$ patients in the tumor bed field group had DM. Also, analysis of LRR-free survival and DM-free 
Table 2 LRR site

\begin{tabular}{|c|c|c|c|}
\hline Recurrence site & T-shape field ( $\mathrm{n}=49), \mathrm{n}(\%)$ & Tumor bed field ( $\mathrm{n}=47), \mathrm{n}(\%)$ & $P$ value \\
\hline Mediastinal lymph nodes & $1(2.04)$ & $7(14.89)$ & $0.049^{c}$ \\
\hline Anastomotic site & $2(4.08)$ & $1(2.13)$ & $1.000^{\mathrm{C}}$ \\
\hline Tumor bed & $1(2.04)$ & $1(2.13)$ & $1.000^{c}$ \\
\hline
\end{tabular}

${ }^{\mathrm{c}}$, Fisher's exact test. LRR, locoregional recurrence.
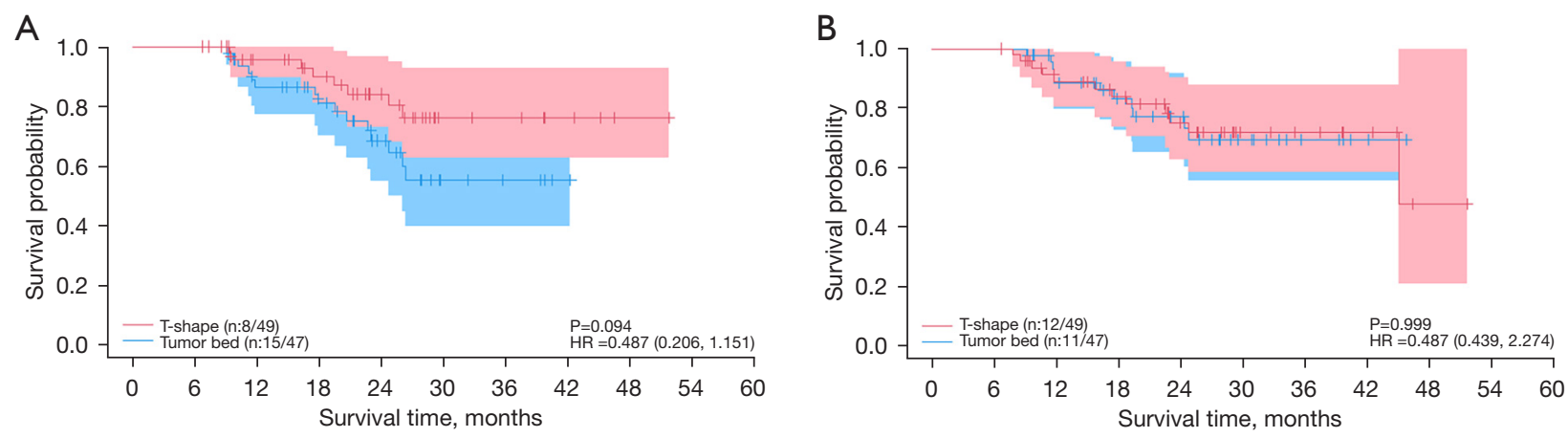

Figure 2 Plot of the LRFS (A) and DMFS (B) of the patients. The HR and the P value are shown. LRFS, locoregional recurrence-free survival; DMFS, distant metastasis-free survival; HR, hazard ratio.

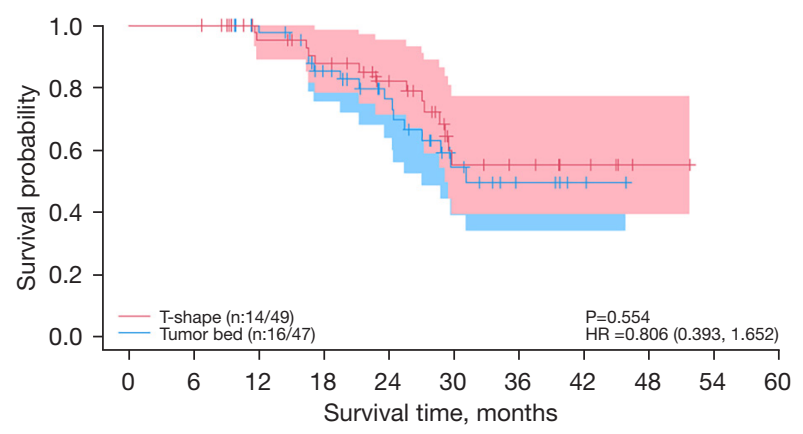

Figure 3 Kaplan-Meier estimates of the OS. The HR and the P value are shown. OS, overall survival; HR, hazard ratio.

survival did not show any statistical difference (Figure 2). Although the OS of the small T-shaped field group (71.4\%) was higher than that of the tumor bed field group (66.0\%), this trend did not reach statistical significance (Figure 3).

For patients in the small T-shaped field group, the mean dose $\left(\mathrm{D}_{\text {mean }}\right)$ to the lung was $11.03 \pm 1.69 \mathrm{~Gy}$ and the lung volume percentage receiving >20 Gy (V20) was $19.95 \% \pm 3.99 \%$, while that receiving $>5$ Gy (V5) was
$47.59 \% \pm 9.43 \%$. As shown in Table 3, we did not observe statistical differences in the risk of grade 2 esophagitis and grade 2 pneumonitis between the two groups. Also, none of the patients suffered $\geq$ grade 3 esophagitis, esophageal stricture, or life-threatening hemorrhage (Table 4).

\section{Discussion}

For locally advanced ESCC patients, surgery alone usually cannot completely remove malignant cells, and thus, a comprehensive postoperative treatment is necessary for reducing the incidence rates of LRR and DM. The issue of optimal CTV range for postoperative radiotherapy in ESCC patients has been a matter of growing concern (23). In the present study, postoperative radiotherapy of the small T-shaped field, which covered tumor bed, positive lymph node areas, bilateral supraclavicular areas, as well as the upper and middle mediastinal lymphatic drainage areas, was used to treat locally advanced ESCC patients, and our results revealed that LRR in the mediastinal lymph nodes of the small T-shaped field group was significantly lower than that of tumor bed field group. At the end of the follow-up 
Table 3 Lung radiation dose

\begin{tabular}{lccc}
\hline Parameter & V5 & V20 & $D_{\text {mean }}$ \\
\hline Lung radiation dose (Gy), mean $\pm S D$ & $47.59 \pm 9.43$ & $19.95 \pm 3.99$ & $11.03 \pm 1.69$ \\
\hline$D_{\text {mean }}$, mean dose. & & &
\end{tabular}

Table 4 Toxicity

\begin{tabular}{|c|c|c|c|c|}
\hline Toxicity & T-shape field ( $n=49), n(\%)$ & Tumor bed field $(n=47), n(\%)$ & Test value & $P$ value \\
\hline Grade 2 pneumonitis & $11(22.45)$ & $8(17.02)$ & 0.158 & $0.341^{\mathrm{a}}$ \\
\hline Grade 3 pneumonitis & 0 & 0 & & \\
\hline Esophageal stricture & 0 & 0 & & \\
\hline
\end{tabular}

${ }^{\mathrm{a}}$, Chi-square test.

period in this study, the OS rate of the small T-shaped field group was higher than that of control group, although this did not reach statistical difference. Therefore, the small T-shaped field might be a safe and effective approach for the postoperative radiotherapy of ESCC patients.

Currently, there is no established consensus on the target volume delineation for postoperative radiotherapy in ESCC patients. The recurrent laryngeal nerves in the superior mediastinum and the bilateral supraclavicular area are closely connected to the surrounding lymph nodes, which greatly increases the difficulty of the operation and may cause the omission of lymph nodes during lymph node lymphadenectomy. In order to gain the maximum benefits from radiotherapy, the areas of CTV should be limited to high-risk regions after radical esophagectomy. Therefore, the recurrence pattern of regional lymph nodes after surgery is a key factor to guide CTV delineation. Studies have shown that the recurrence of upper and middle thoracic esophageal carcinoma after radical surgery often occurs in the bilateral supraclavicular areas and upper mediastinum (5,24-26). Similar to upper and middle thoracic esophageal carcinoma, upper mediastinal lymph node dissection is more difficult for lower esophageal cancer due to the complex anatomical structure. Multiple studies have shown that bilateral supraclavicular and superior mediastinal areas have the highest recurrence rates in patients with lower thoracic ESCC $(24,25)$. It has been reported that $79.4 \%$ of all the recurrent sites are located in either the bilateral supraclavicular areas or the upper mediastinum (5). A retrospective study involving 244 patients showed that the most common areas of recurrence were the lymph nodes in the lower neck, as well as the middle and upper mediastinum (27). The most common sites of regional lymph node metastasis are the upper mediastinum $(73.8 \%)$, followed by the supraclavicular (43.7\%), middle mediastinum (39.7\%), abdominal cavity (13.5\%), and inferior mediastinum (1.6\%) after radical surgery with two-field lymph node dissection (25). Furthermore, it has been suggested that irradiation of the upper abdomen area is unnecessary, because no patients experience failure in the abdomen regardless of whether or not they receive postoperative radiation (21). Therefore, we designed the small T-shaped field for postoperative radiotherapy of ESCC patients by using IMRT technique to cover the above-mentioned high-risk recurrence areas. The effectiveness and therapy-related toxicity of the small T-shaped field was compared to that of tumor bed field, and statistical analysis revealed the benefits in terms of the LRR rates of the mediastinal lymph nodes.

Acute radiation pneumonitis is one of the main doselimiting factors for thoracic radiotherapy (28). V20 and $\mathrm{D}_{\text {mean }}$ to the lung are currently recognized as dosimetry parameters related to radiation pneumonitis (29). With the widespread application of IMRT technology, the volume of the high-dose area of the lung can be effectively reduced; however, due to factors such as the increase of the radiation field and the ray scattering between the gratings, the lowdose area also increases compared with two-dimensional radiotherapy technology. A previous retrospective analysis revealed that the lung V5 and V20 were independent predictors for radiation pneumonitis above grade 2 (30). 
Moreover, lower lobe exposure to radiation seems to be another determinant for radiation pneumonitis $(31,32)$, and this dissimilar regional radiosensitivity might be related to better oxygenation, perfusion, and ventilation of the lower lobes (33). In this study, the dose parameters of the small T-shaped field were markedly lower than the threshold that could induce radiation pneumonitis, and few lower pulmonary regions were encompassed, thus no acute radiation pneumonitis (grade 3 or above) was observed. Since the irradiated volume was greater in the small T-shaped field, the incidence of grade 2 radiation pneumonitis and grade 2 radiation esophagitis was higher compared to the tumor bed field group, although the differences were not statistically significant. These adverse reactions were tolerable and controllable in all patients.

In conclusion, treatment failures after radical esophagectomy of ESCC involve LRR and DM. Accumulating evidence indicates that LRR in the upper and middle mediastinum and supraclavicular accounts for a considerable proportion. Based on these facts, we treated ESCC patients with small T-shaped field postoperative radiotherapy and demonstrated the feasibility of this approach. The limitation of the current study is that the sample size of the retrospective analysis was relatively small, and therefore, follow-up randomized controlled investigations with a larger sample sizes are required to further validate the efficacy and safety of this approach.

\section{Acknowledgments}

Funding: None.

\section{Footnote}

Reporting Checklist: The authors have completed the STROBE reporting checklist. Available at https://dx.doi. org/10.21037/jgo-21-604

Data Sharing Statement: Available at https://dx.doi. org/10.21037/jgo-21-604

Conflicts of Interest: All authors have completed the ICMJE uniform disclosure form (available at https://dx.doi. org/10.21037/jgo-21-604). The authors have no conflicts of interest to declare.

Ethical Statement: The authors are accountable for all aspects of the work in ensuring that questions related to the accuracy or integrity of any part of the work are appropriately investigated and resolved. The study was conducted in accordance with the Declaration of Helsinki (as revised in 2013). The study was approved by ethics board of The First Affiliated Hospital of Soochow University and informed consent was taken from all the patients.

Open Access Statement: This is an Open Access article distributed in accordance with the Creative Commons Attribution-NonCommercial-NoDerivs 4.0 International License (CC BY-NC-ND 4.0), which permits the noncommercial replication and distribution of the article with the strict proviso that no changes or edits are made and the original work is properly cited (including links to both the formal publication through the relevant DOI and the license). See: https://creativecommons.org/licenses/by-nc-nd/4.0/.

\section{References}

1. Torre LA, Bray F, Siegel RL, et al. Global cancer statistics, 2012. CA Cancer J Clin 2015;65:87-108.

2. Stewart BW, Wild CP, Stewart BW. editors. World cancer report 2014. Geneva: World Health Organization, 2015.

3. Kumagai K, Rouvelas I, Tsai JA, et al. Meta-analysis of postoperative morbidity and perioperative mortality in patients receiving neoadjuvant chemotherapy or chemoradiotherapy for resectable oesophageal and gastrooesophageal junctional cancers. Br J Surg 2014;101:321-38.

4. Chen G, Wang Z, Liu XY, et al. Recurrence pattern of squamous cell carcinoma in the middle thoracic esophagus after modified Ivor-Lewis esophagectomy. World J Surg 2007;31:1107-14.

5. Liu Q, Cai XW, Wu B, et al. Patterns of failure after radical surgery among patients with thoracic esophageal squamous cell carcinoma: implications for the clinical target volume design of postoperative radiotherapy. PLoS One 2014;9:e97225.

6. Chen J, Pan J, Liu J, et al. Postoperative radiation therapy with or without concurrent chemotherapy for nodepositive thoracic esophageal squamous cell carcinoma. Int J Radiat Oncol Biol Phys 2013;86:671-7.

7. Chen J, Zhu J, Pan J, et al. Postoperative radiotherapy improved survival of poor prognostic squamous cell carcinoma esophagus. Ann Thorac Surg 2010;90:435-42.

8. Wang ZW, Luan ZP, Zhang W, et al. Postoperative chemoradiotherapy improves survival in esophageal squamous cell cancer with extracapsular lymph node extension. Neoplasma 2014;61:732-8. 
9. Chen HS, Wu SC, Hsu PK, et al. The prognostic impact of preoperative and postoperative chemoradiation in clinical stage II and III esophageal squamous cell carcinomas: a population based study in Taiwan. Medicine (Baltimore) 2015;94:e1002.

10. Wang $S$, Wang $Z$, Yang $Z$, et al. Postoperative Radiotherapy Improves Survival in Stage pT2N0M0 Esophageal Squamous Cell Carcinoma with High Risk of Poor Prognosis. Ann Surg Oncol 2016;23:265-72.

11. Huang W, Li B, Gong H, et al. Pattern of lymph node metastases and its implication in radiotherapeutic clinical target volume in patients with thoracic esophageal squamous cell carcinoma: a report of 1077 cases. Radiother Oncol 2010;95:229-33.

12. Wang X, Luo Y, Li M, et al. Recurrence pattern of squamous cell carcinoma in the midthoracic esophagus: implications for the clinical target volume design of postoperative radiotherapy. Onco Targets Ther 2016;9:6021-7.

13. Xiao ZF, Yang ZY, Liang J, et al. Value of radiotherapy after radical surgery for esophageal carcinoma: a report of 495 patients. Ann Thorac Surg 2003;75:331-6.

14. Bollschweiler E, Schröder W, Hölscher AH, et al. Preoperative risk analysis in patients with adenocarcinoma or squamous cell carcinoma of the oesophagus. Br J Surg 2000;87:1106-10.

15. Tachibana M, Dhar DK, Kinugasa S, et al. Esophageal cancer with distant lymph node metastasis: prognostic significance of metastatic lymph node ratio. J Clin Gastroenterol 2000;31:318-22.

16. Prenzel KL, Bollschweiler E, Schröder W, et al. Prognostic relevance of skip metastases in esophageal cancer. Ann Thorac Surg 2010;90:1662-7.

17. Cai XW, Zeng Y, Feng W, et al. Randomized phase II trial comparing tumor bed alone with tumor bed and elective nodal postoperative radiotherapy in patients with locoregionally advanced thoracic esophageal squamous cell carcinoma. Dis Esophagus 2019;32:doz013.

18. Ténière P, Hay JM, Fingerhut A, et al. Postoperative radiation therapy does not increase survival after curative resection for squamous cell carcinoma of the middle and lower esophagus as shown by a multicenter controlled trial. French University Association for Surgical Research. Surg Gynecol Obstet 1991;173:123-30.

19. Qiao XY, Wang W, Zhou ZG, et al. Comparison of efficacy of regional and extensive clinical target volumes in postoperative radiotherapy for esophageal squamous cell carcinoma. Int J Radiat Oncol Biol Phys 2008;70:396-402.
20. Xiao ZF, Yang ZY, Miao YJ, et al. Influence of number of metastatic lymph nodes on survival of curative resected thoracic esophageal cancer patients and value of radiotherapy: report of 549 cases. Int J Radiat Oncol Biol Phys 2005;62:82-90.

21. Chen J, Pan J, Zheng X, et al. Number and location of positive nodes, postoperative radiotherapy, and survival after esophagectomy with three-field lymph node dissection for thoracic esophageal squamous cell carcinoma. Int J Radiat Oncol Biol Phys 2012;82:475-82.

22. Rice TW, Ishwaran H, Hofstetter WL, et al. Recommendations for pathologic staging (pTNM) of cancer of the esophagus and esophagogastric junction for the 8th edition AJCC/UICC staging manuals. Dis Esophagus 2016;29:897-905.

23. Zhu Y, Li M, Kong L, et al. Postoperative radiation in esophageal squamous cell carcinoma and target volume delineation. Onco Targets Ther 2016;9:4187-96.

24. Wang Y, Zhang L, Ye D, et al. A retrospective study of pattern of recurrence after radical surgery for thoracic esophageal carcinoma with or without postoperative radiotherapy. Oncol Lett 2018;15:4033-9.

25. Li CL, Zhang FL, Wang YD, et al. Characteristics of recurrence after radical esophagectomy with two-field lymph node dissection for thoracic esophageal cancer. Oncol Lett 2013;5:355-9.

26. Doki Y, Ishikawa O, Takachi K, et al. Association of the primary tumor location with the site of tumor recurrence after curative resection of thoracic esophageal carcinoma. World J Surg 2005;29:700-7.

27. Cai WJ, Xin PL. Pattern of relapse in surgical treated patients with thoracic esophageal squamous cell carcinoma and its possible impact on target delineation for postoperative radiotherapy. Radiother Oncol 2010;96:104-7.

28. Jain V, Berman AT. Radiation pneumonitis: old problem, new tricks. Cancers (Basel) 2018;10:222.

29. Hanania AN, Mainwaring W, Ghebre YT, et al. Radiationinduced lung injury: assessment and management. Chest 2019;156:150-62.

30. Shaikh T, Churilla TM, Monpara P, et al. Risk of radiation pneumonitis in patients receiving taxane-based trimodality therapy for locally advanced esophageal cancer. Pract Radiat Oncol 2016;6:388-94.

31. Bradley JD, Hope A, El Naqa I, et al. A nomogram to predict radiation pneumonitis, derived from a combined analysis of RTOG 9311 and institutional data. Int J Radiat Oncol Biol Phys 2007;69:985-92. 
32. Katsui K, Ogata T, Watanabe K, et al. Dose-volume parameters predict radiation pneumonitis after induction chemoradiotherapy followed by surgery for non-small cell lung cancer: a retrospective analysis. BMC Cancer 2019;19:1144.

Cite this article as: Zhao Q, Zhu J, Liu Y, Qin S, Zhou J. Preliminary evaluation of postoperative radiotherapy with small T-shaped field in thoracic esophageal squamous cell carcinoma. J Gastrointest Oncol 2021;12(5):2004-2012. doi: 10.21037/jgo21-604
33. Kong FM, Wang S. Nondosimetric risk factors for radiation-induced lung toxicity. Semin Radiat Oncol 2015;25:100-9.

(English Language Editor: A. Kassem) 